\title{
The anomalous chromomagnetic dipole moment of the top quark in the standard model and beyond
}

\author{
R. Martinez and J-Alexis Rodriguez闭 \\ Departamento de Física, Universidad Nacional de Colombia \\ Bogota, Colombia
}

\begin{abstract}
The anomalous chromomagnetic moment of the top quark arises from one loop corrections to the vertex $\bar{t} t g$. We give explicit formulae for this anomalous coupling in the framework of the standard model, the two Higgs doublet model and the minimal supersymmetric standard model. We compare the results for the anomalous coupling with the bound $-0.03 \leq \Delta \kappa \leq 0.01$ emerging from the analysis of $b \rightarrow s \gamma$ process with an on-shell bremsstrahlung gluon. This enables us to study the allowed region of parameters of the models under consideration.
\end{abstract}

The top quark is the heaviest fermion in the standard model (SM) with a mass of $174 \pm 5.2 \mathrm{GeV}$. Soon detailed experimental studies of the top quark properties, will be available. This will give us the envisaged high precision of data opening a rich field of new top quark phenomenology. In the framework of the SM, the couplings of the top quark are fixed by the gauge symmetry, the structure of generations and the dimension of the interaction Lagrangian. This makes the top quark an ideal candidate to search for new physics beyond the SM. Anomalous couplings between the top quark and gauge bosons might affect the top quark production at high energies and also its decay rate. Precisely measured quantities with virtual top quark contributions [1, 2, 3] will yield further information regarding these couplings.

Modifications of the SM couplings can be traced back to the dimension of the operators in the effective Lagrangian description valid above the electroweak symmetry breaking scale. They would be, in principle, of the same order as the other dimension 5 and 6 couplings below the electroweak scale. By means of the dimension 5 coupling to an on-shell gluon, the anomalous chromomagnetic dipole moment of the top quark is defined as

$$
L_{5}=i\left(\frac{\Delta \kappa}{2}\right) \frac{g_{s}}{2 m_{t}} \bar{u}(t) \sigma_{\mu \nu} q^{\nu} T^{a} u(t) G^{\mu, a}
$$

where $g_{s}$ and $T^{a}$ are the $S U(3)_{c}$ coupling and generators, respectively, and the parameter $\Delta \kappa$ is identified with the anomalous chromomagnetic dipole moment of the top quark.

The effects due to $\Delta \kappa \neq 0$ were examined in flavor physics as well as high top quark cross section measurements [1, 2, 3, 4, 5]. In the latter case, the parton level differential cross sections of $g g \rightarrow \bar{t} t$ and $\bar{q} q \rightarrow \bar{t} t$ (the dominant channel at Tevatron energies) were calculated [3, 4, 6]. The combined effects of the chromomagnetic and the chromoelectric dipole moment of the top quark on the reaction $p \bar{p} \rightarrow t \bar{t} X$ were investigated in reference [2]. Moreover, previous analysis has revealed that the

\footnotetext{
*romart@ciencias.unal.edu.co

†alexro@ciencias.unal.edu.co
}

differential cross section is sensitive to the sign of the anomalous chromomagnetic dipole moment on account of the interference with the SM coupling.This can lead to a significant suppression or enhancement in the production rate [3].

We mention that on the one hand the $\bar{t} t g$ coupling correction to the total top quark production cross section at Fermilab was calculated by Stange et.al [4]. In the framework of the SM, they found that the correction is less than $2.4 \%$ which is much less than the uncertainty in the cross section. Also, they found that in the general two Higgs doublet model (2HDM), corrections can be significantly enhanced, and can be as large as $20 \%$. On the other hand, for the minimal supersymmetric standard model (MSSM) the corrections of the Higgs sector never exceed $20 \%$. Finally, in connection to the cross section of the top quark production, the genuine supersymmetric electroweak corrections to $\bar{t} t g$ vertex were calculated in [7, 8]. The result of this calculation is that for $\tan \beta>1$ and the bottom-squark mass $<150 \mathrm{GeV}$ the correction can exceed 20\%. Reference [9] evaluated the supersymmetric QCD corrections to single top quark production and found that the combined effects of supersymmetry and SM might reach the $10 \%$ for small $\tan \beta$.

It has been pointed out that $\Delta \kappa$ would be more easily probed at the LHC than the Tevatron. Roughly speaking, the sensitivity of LHC can be a factor of three better getting close to values of $\Delta \kappa$ as small as 0.03 [3, 6]. NonQCD radiative corrections on the $t \bar{t}$ production at the LHC amounts to $2.5 \%$ including the SM with a Higgs boson mass $\sim 100 \mathrm{GeV}$. For the general 2HDM the contribution of radiative corrections is less than $4 \%$, for SUSY electroweak one-loop corrections less than $10 \%$ and if only SUSY QCD one-loop corrections are considered, about $4 \%$ [10].

Since the anomalous chromomagnetic dipole moment of the top quark appears in the top quark cross section, it is possible, due to uncertainties, to estimate the constraints that it would impose on the $\Delta \kappa$. For the LHC, the anomalous coupling is constrained to lie in the range $-0.09 \leq \Delta \kappa \leq 0.1$ [3]. Similar range is obtained for the future NLC. The influence of an anomalous $\Delta \kappa$ on the cross section and associated gluon jet energy for $t \bar{t} g$ has been also analyzed. Events produced at $500 \mathrm{GeV}$ in 
$e^{+} e^{-}$colliders, with a cut on the gluon energy of $500 \mathrm{GeV}$ and integrated luminosity of $30 \mathrm{fb}^{-1}$, lead to a bound of $-2.1 \leq \Delta \kappa \leq 0.6$. Finally in this context, Rizzo [3] showed that the top quark $p_{t}$ and $M_{t t}$ distributions are the most sensitive observables to non-zero values of $\Delta \kappa$.

From the experimental information it is possible to get a limit on the $\Delta \kappa$ from Tevatron. Following the reference by F. del Aguila [11] and assuming that the only non-zero coupling is precisely the chromomagnetic dipole moment of the top quark, we find from the collected data that the allowed region is $|\Delta \kappa| \leq 0.45$.

We call to the attention that although the vertex $t t g$ is involved in the top quark pair production, the anomalous factor would be measured at transferred momentum different from zero which, of course means an off-shell gluon. Anomalous couplings of the top quark to on-shell gluons would modify the rate for $B \rightarrow X_{s} \gamma$ [12, 13. The presence of the magnetic dipole moment would affect the Wilson coefficients which mediate $b \rightarrow s$ transitions by the coefficients $C_{7,8}$ of the one-loop matching conditions for the magnetic and chromomagnetic dipole operators $O_{7,8}$. Comparing the calculated branching fraction which involves the anomalous coupling, to the CLEO measurements it is possible to get an allowed region for this anomalous coupling [12, 13. Using the recent data from CLEO collaboration for the branching fraction of the process $B(b \rightarrow s \gamma)=(3.21 \pm 0.43 \pm 0.27) \times 10^{-4}$ [14, we update the previous analysis done in reference 113 and get a new allowed region for the anomalous chromomagnetic dipole moment of the top quark to be $-0.03 \leq \Delta \kappa \leq 0.01$.

Our objective in this paper is to evaluate the contribution at the one loop-level to the anomalous chromomagnetic dipole moment of the top quark in different scenarios with the gluon boson on-shell. We study the region of allowed parameter space in different frameworks. We derive our bounds on $\Delta \kappa$ from the analysis performed for the $b \rightarrow s \gamma$ process [13].

Beginning with the SM, the typical QCD correction through gluon exchange implies two different Feynman diagrams: the first one is equivalent to the QED contribution where the external gluon is coupled to the fermion line in the loop and in the second one the external gluon is coupled to internal gluons due to the non-abelian character of the $S U(3)$ color group. After the explicit calculation of the loops, we find that the second diagram does not contribute and the final result is,

$$
\Delta \kappa=-\frac{1}{6} \frac{\alpha_{s}\left(m_{t}\right)}{\pi}
$$

We note that its natural size is of the order of $\alpha_{s} / \pi$ similar to the QED anomalous coupling, but now in combination with a factor $-1 / 6$ coming from the color structure in the diagram i.e. $T^{a} T^{b} T^{a}=-T^{b} / 6$ with $T^{a}$ being the generators of $S U(3)_{C}$.

The other possible contribution in the framework of the SM comes from electroweak interactions. The relevant contributions occur when neutral Higgs boson and the

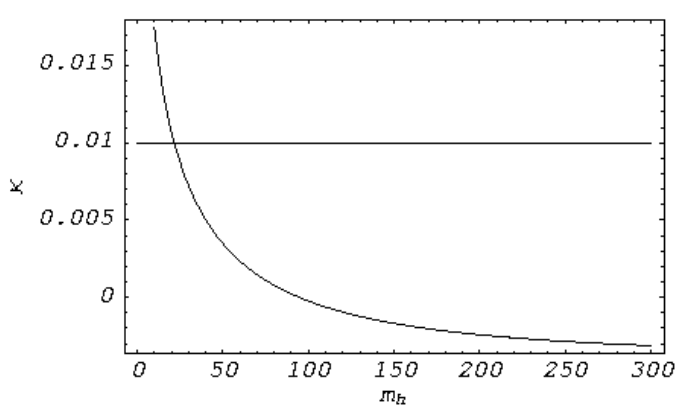

FIG. 1: Standard Model contribution to the anomalous chromomagnetic dipole moment of the top quark versus the Higgs boson mass.

would-be Goldstone boson of $Z$ are involved in the loop. This contribution reads

$$
\Delta \kappa=-\frac{\sqrt{2} G_{F} m_{t}^{2}}{8 \pi^{2}}\left[H_{1}\left(m_{h}\right)+H_{2}\left(m_{Z}\right)\right],
$$

where

$$
\begin{aligned}
& H_{1}(m)=\int_{0}^{1} d x \frac{x-x^{3}}{x^{2}-\left(2-m^{2} / m_{t}^{2}\right) x+1}, \\
& H_{2}(m)=\int_{0}^{1} d x \frac{-x+2 x^{2}-x^{3}}{x^{2}-\left(2-m^{2} / m_{t}^{2}\right) x+1} .
\end{aligned}
$$

This expression agree with similar one presented in reference 15. The SM contribution is showed in figure 1 where we have added the QCD contribution (2). It is worth noting that the behaviour of the curve for a large Higgs boson mass indicates decoupling and that the values of $\Delta \kappa$ lie within the allowed region for $\Delta \kappa$ coming from $b \rightarrow s \gamma$.

The contributions within a general $2 \mathrm{HDM}$ will be different from the SM contributions because of the presence of the virtual five physical Higgs bosons which appear in any two Higgs doublet model after spontaneous symmetry breaking: $H^{0}, A^{0}, h^{0}, H^{ \pm}$16. Therefore, 2HDM predictions depend on their massses and on the two mixing angles $\alpha$ and $\beta$. For small $\beta$, the charged Higgs boson

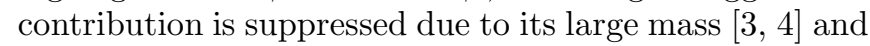
the small bottom quark mass.

The expression for the contribution of the neutral Higgs bosons is given by

$$
\begin{aligned}
\Delta \kappa & =\frac{\sqrt{2} G_{F}}{8 \pi^{2}}\left[\lambda_{H^{0} t t}^{2} H_{1}\left(M_{H}^{2}\right)+\lambda_{h^{0} t t}^{2} H_{1}\left(M_{h}^{2}\right)\right. \\
& \left.+\lambda_{A^{0} t t}^{2} H_{2}\left(M_{A}^{2}\right)+\lambda_{G^{0} t t}^{2} H_{2}\left(M_{Z}^{2}\right)\right]
\end{aligned}
$$

where $\lambda_{i t t}$ are the Yukawa couplings in the so-called models of type I, II and III [16]. Table I shows the couplings in the usual convention.

The Yukawa couplings of a given fermion to the Higgs scalars are proportional to the mass of the fermion and they are therefore naturally enhanced in this case. In 


\begin{tabular}{lccc}
\hline$\lambda_{i t t}$ & model type I & model type II & model type III \\
\hline$\lambda_{H^{0} t t}$ & $\frac{m_{t} \sin \alpha}{\sin \beta}$ & $\frac{m_{t} \cos \alpha}{\cos \beta}$ & $\left(1+\frac{\eta_{t t}^{U}}{\sqrt{2}}\right) m_{t} \sin \alpha$ \\
$\lambda_{h^{0} t t}$ & $\frac{m_{t} \cos \alpha}{\sin \beta}$ & $\frac{m_{t} \sin \alpha}{\cos \beta}$ & $\left(1+\frac{\eta_{t t}^{U}}{\sqrt{2}}\right) m_{t} \cos \alpha$ \\
$\lambda_{A^{0} t t}$ & $\cot \beta m_{t}$ & $\tan \beta m_{t}$ & $\frac{\eta_{t t}^{U} m_{t}}{\sqrt{2}}$ \\
$\lambda_{G^{0} t t}$ & $m_{t}$ & $m_{t}$ & $m_{t}$ \\
\hline
\end{tabular}

TABLE I: Couplings of the Higgs eigenstates with the top quark which are relevant in the calculation of the anomalous chromomagnetic dipole moment of the top quark in 2HDM in its different versions. We omit the factor $g / 2 m_{W}$ and in the model type III we use the Sher-Cheng approach for the flavour changing couplings, hence the parameters $\eta_{i j} \sim 1$ for the numerical analysis 17 .

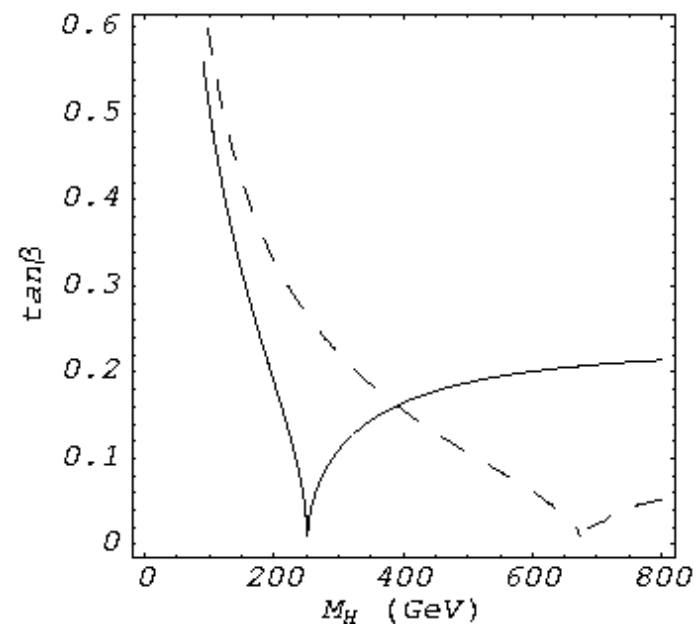

FIG. 2: Contour plot for the contribution of $2 \mathrm{HDM}$ to the anomalous chromomagnetic dipole moment of the top quark in the plane $\tan \beta-m_{H}$ for $m_{A}=200 \mathrm{GeV}$ (solid line) and $m_{A}=400 \mathrm{GeV}$ (dashed line) using the bound from the $b \rightarrow s \gamma$ process. The allowed region is above the curve.

the model type III appears flavour changing neutral couplings at tree level which can be parametrized in the SherCheng approach where a natural value for the flavour changing couplings from different families should be of the order of the geometric average of their Yukawa couplings, $h_{i j}=g \eta_{i j} \sqrt{m_{i} m_{j}} /\left(2 m_{W}\right)$ with $\eta_{i j}$ of the order of one 17.

In order to show the behaviour of the contribution of the 2HDM to the anomalous chromomagnetic dipole moment of the top quark, we evaluate explicitly the contribution for couplings type II. We show in figure
2 the allowed region (above the curve) for the plane $\tan \beta$ vs $m_{H}$ using equation (4) and assuming that $\Delta \kappa$ is $-0.03 \leq \Delta \kappa \leq 0.01$ from $b \rightarrow s \gamma \sqrt{13}]$. We fix the following parameters: $m_{H}=m_{h}, m_{A}=200(400) \mathrm{GeV}$ solid line (dashed line). The solid line for the scalar Higgs mass smaller (bigger) than $240 \mathrm{GeV}$ corresponds to the cut between equation (4) and the upper(lower) limit from $b \rightarrow s \gamma$. In figure 3 as in figure 2 , we display the allowed region for the plane $\tan \beta$ vs $m_{A}$ with $m_{H}=m_{h}=90(200) \mathrm{GeV}$ for the solid (dashed) line. In this case we only find cuts with the upper limit for $\Delta \kappa$ from $b \rightarrow s \gamma$.

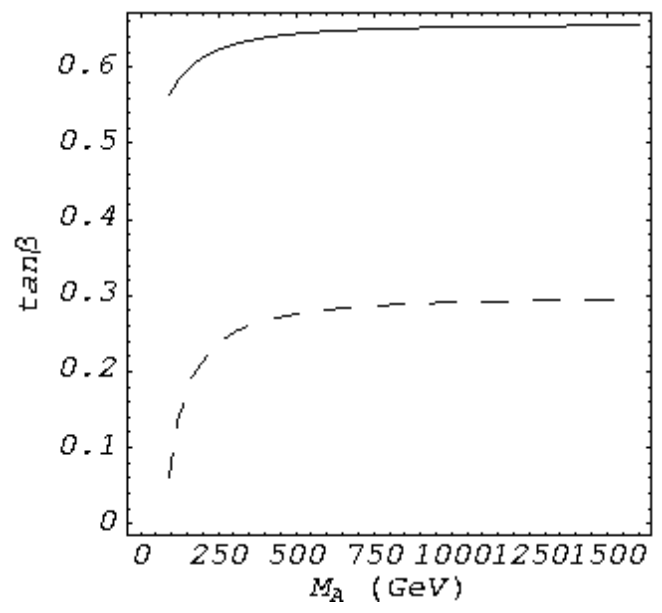

FIG. 3: Contour plot for the plane $\tan \beta-m_{A}$ with the common scalar mass $m_{h}=m_{H}=90(200) \mathrm{GeV}$ for the solid line (dashed line). The allowed region is above the curve.

Our last step is to calculate the anomalous chromomagnetic dipole moment of the top quark in the framework of the MSSM. We only consider the SUSY QCD contribution which is generated from the exchange of gluinos and squarks. The mixing in the squark sector in this model is particularly relevant in the case of the squark top because the top quark mass is involved. In this case is possible to neglect the family mixing and consider only the mixing between the superpartners for the left- and right handed top quark. The mass eigenstates are given by

$$
\tilde{t}_{a}=\sum_{b=i j} R_{a b} \tilde{q_{b}}
$$

with $R_{a b}$ the rotation matrix which diagonalizes the stop mass matrix

$$
M_{\tilde{t}}=\left(\begin{array}{cc}
\tilde{M}_{Q}+m_{Z}^{2} \cos 2 \beta\left(\frac{1}{2}-\frac{2}{3} \sin ^{2} \theta_{w}\right)+m_{t}^{2} & \left(\mu \cot \beta+A_{t} \tilde{M}\right) m_{t} \\
\left(\mu \cot \beta+A_{t} \tilde{M}\right) m_{t} & \tilde{M}_{U}+m_{Z}^{2} \cos 2 \beta\left(\frac{2}{3} \sin ^{2} \theta_{w}\right)+m_{t}^{2}
\end{array}\right)
$$


where $\tilde{M}_{Q, U}$ are the soft SUSY breaking terms, $\mu$ is the coefficient of the bilinear Higgs term and $A_{t}$ is the trilinear soft SUSY breaking parameter. In this case, the mixing angle and the mass eigenstates are

$$
\begin{aligned}
\tan 2 \theta_{t} & =\frac{2\left(\mu \cot \beta+A_{t} \tilde{M}\right) m_{t}}{\Delta \tilde{m}_{t}} \\
\tilde{m}_{t_{1,2}} & =\frac{1}{2}\left[\tilde{m}_{t_{L}}^{2}+\tilde{m}_{t_{R}}^{2} \pm \sqrt{\left(\Delta \tilde{m}_{t}\right)^{2}+4\left(\mu \cot \beta+A_{t} \tilde{M}\right)^{2} m_{t}^{2}}\right]
\end{aligned}
$$

where $\Delta \tilde{m}_{t}=\tilde{m}_{t_{L}}^{2}-\tilde{m}_{t_{R}}^{2}$ and $\tilde{m}_{t_{L,(R)}}^{2}$ are the diagonal entries in the matrix (6).

In this framework the SUSY QCD contribution arises from the exchange of $\tilde{t}_{1,2}$ and gluinos. The contribution for the virtual $\tilde{t}_{1}$ turns out to be

$$
\begin{aligned}
\Delta \kappa & =\frac{4 \alpha_{s}}{3 \pi}\left[\cos ^{2} \theta_{t} H_{3}\left(\hat{m}_{g}, \hat{\tilde{m}}_{t}\right)-\sin ^{2} \theta_{t} H_{4}\left(\hat{m}_{g}, \hat{\tilde{m}}_{t}\right)+\hat{m}_{g} \sin \theta_{t} \cos \theta_{t} H_{5}\left(\hat{m}_{g}, \hat{\tilde{m}}_{t}\right)\right] \\
& +\frac{3 \alpha_{s}}{2 \pi}\left[\cos ^{2} \theta_{t} H_{3}\left(\hat{\tilde{m}}_{t}, \hat{m}_{g}\right)+\sin ^{2} \theta_{t} H_{4}\left(\hat{\tilde{m}}_{t}, \hat{m}_{g}\right)-\hat{m}_{g} \sin \theta_{t} \cos \theta_{t} H_{5}\left(\hat{\tilde{m}}_{t}, \hat{m}_{g}\right)\right]
\end{aligned}
$$

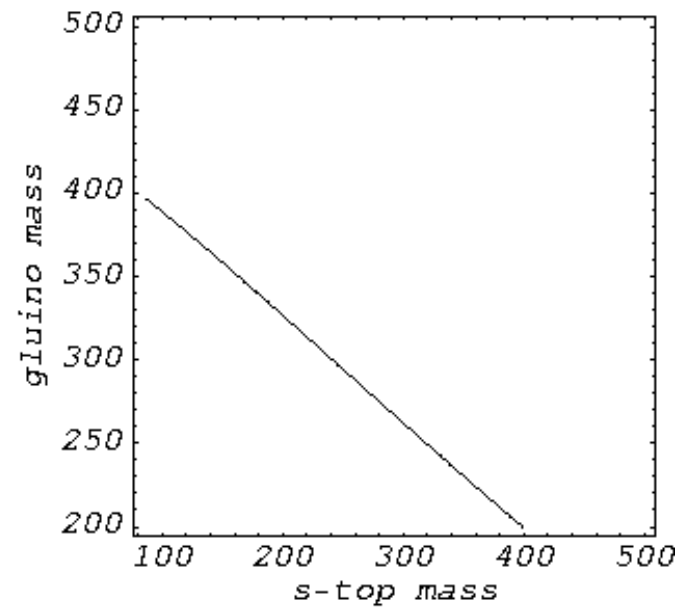

FIG. 4: Contour plot for the supersymmetric QCD contribution to the anomalous chromomagnetic dipole moment of the top quark using the bound from the $b \rightarrow s \gamma$ process. The allowed region is above the curve.

with the functions

$$
\begin{aligned}
& H_{3}\left(m_{1}, m_{2}\right)=\int_{0}^{1} d x \frac{x^{2}-3 x^{3} / 2}{x^{2}-\left(1-m_{1}^{2}+m_{2}^{2}\right)+m_{2}^{2}} \\
& H_{4}\left(m_{1}, m_{2}\right)=\int_{0}^{1} d x \frac{x^{3} / 2}{x^{2}-\left(1-m_{1}^{2}+m_{2}^{2}\right)+m_{2}^{2}} \\
& H_{5}\left(m_{1}, m_{2}\right)=\int_{0}^{1} d x \frac{x^{2}-x}{x^{2}-\left(1-m_{1}^{2}+m_{2}^{2}\right)+m_{2}^{2}}(10)
\end{aligned}
$$

where $\hat{m}=m / m_{t}$. For the squark $\tilde{t}_{2}$ the expression is obtained by changing $\sin \theta_{t}$ to $\cos \theta_{t}$ and vice-versa.

Similar to figure 2 and 3 , the plot in figure 4 refers to the allowed region of the parameter space obtained from constraints on $\Delta \kappa$ from $b \rightarrow s \gamma$. Here the plane is of the stop mass vs gluino mass with the SUSY parameters fixed to $\theta_{t}=0.98$ and $\tilde{m}_{t_{1}}=\tilde{m}_{t_{2}}$. The mixing angle is chosen such that the coupling of the squark top with the $Z^{0}$ boson vanishes and therefore we use the lower limits for the squark mass obtained from the LEP searching [18. The allowed region is above the curve and the solid line corresponds to the cut of the upper limit of the bound of $\Delta \kappa$ coming from $b \rightarrow s \gamma$ and $\Delta \kappa$ from equation (9).

In conclusion, the anomalous chromomagnetic dipole moment of the top quark receives the less stringent bound $|\Delta \kappa| \leq 0.45$ from the Tevatron experiments under the assumption that it is the only non-zero anomalous coupling. On the other hand, we found a more stringent bound from the transition $b \rightarrow s \gamma$, recently measured with improved precision by CLEO collaboration [14], and the bound is $-0.03 \leq \Delta \kappa \leq 0.01$. We have also calculated the chromomagnetic dipole moment of the top quark in the framework of the SM, 2HDM and SUSY-QCD. We found that in SM the anomalous coupling is up to the order of $\pm 10^{-2}$ and it is tending to $-4 \times 10^{-3}$ in the decoupling limit for a large Higgs mass. Furthermore, the anomalous coupling is equal to $-7 \times 10^{-4}$ around a Higgs mass of $113 \mathrm{GeV}$ which is the lower experimental limit from LEP2 18]. If we keep in mind the sensitivity of future and current experiments to $\Delta \kappa(\sim 0.03)$ [3] and its present status, it is clear that an experimental measurement of this anomalous coupling could be a harbinger of physics beyond the SM.

In the $2 \mathrm{HDM}$ the anomalous coupling of the top quark can reach $\sim 10^{-1}$ values, which is also possible for the supersymmetric QCD corrections. We have compared the values of $\Delta \kappa$ obtained in different scenarios with the bound from the chromomagnetic dipole moment of the top quark resulting from $b \rightarrow s \gamma$ process. We have done this, because our considerations apply for on-shell gluons. Therefore, we have presented a parameter analysis for different frameworks which allows an anomalous coupling of the same order that the bound obtained from the $b \rightarrow$ $s \gamma$. The allowed regions for parameters of the 2HDM type II are completely consistent with the unexplored experimental regions for $\tan \beta<1$ coming from analysis using data from LEP [18]. Also, the allowed region for 
the plane $\tan \beta$-gluino mass agree with the experimental bounds.

We acknowledge to M. Nowakowski for the careful reading of the manuscript. This work was supported by COLCIENCIAS, DIB and DINAIN.
[1] K. Cheung, Phys. Rev. D, 53 (1996) 3604.

[2] P. Haberl, O. Nachtmann and A. Wilch, Phys. Rev D,53(1996)4875; T. Rizzo, Phys. Rev. D,53(1996)6218.

[3] D. Atwood, A. Kagan and T. Rizzo, Phys. Rev. D,52(1995)6264; T. G. Rizzo, hep-ph/9902273; T.G. Rizzo, Phys. Rev. D, 50 (1994) 4478.

[4] A.Stange and S. Willenbrock, Phys. Rev. D,48 (1993)2054.

[5] K. Hikasa, Whisnant, Yang and Young, Phys. Rev. D, 58 (1998) 114003.

[6] D. Silverman and G. Shaw, Phys. Rev D ,27 (1983)1196.

[7] J. M. Yang and C. S. Li, Phys. Rev D,52 (1995)1541.

[8] J. M. Yang and C. S. Li, Phys. Rev D,54 (1996)4380; C. S. Li, O. Oakes,J. M. Yang, ibid.,55 (1997)1672.

[9] C. S. Li, H. Y. Zhou, Y. L. Zhu and J. M. Yang, hepph/9606271, hep-ph/97064112; S. Alam, K. Hawigara and S. Matsumoto, Phys. Rev. D,55 (1997)1307.

[10] W. Hollik, W. M. Mosle and D. Wackeroth, Nucl. Phys. B,516 (1998) 29.
[11] F. Abe, et.al, Phys. Rev. Lett.,80 (1998) 2525; T. Han, K. Whisnant, B. L. Young, and X. Zhang, Phys. Lett. B, 385 (1996) 311; F.de Aguila, hep-ph/9911399.

[12] J.L Hewett and T. G. Rizzo, Phys. Rev. D, 49 (1994) 319; J. L. Hewett, Int. J. Mod. Phys. A, 13 (1998)2389.

[13] R. Martinez and J.-Alexis Rodriguez, Phys. Rev D, 55 (1997)3212.

[14] CLEO collaboration, hep-ex/0108032.

[15] R.D. Peccei and X. Zhang, Nucl. Phys. B 337, 269 (1990); R.D. Peccei, S. Peris and X. Zhang, Nucl. Phys. B 349, 305 (1991).

[16] Dawson, Gunion, Haber, Kane, The Higgs Hunter's Guide, Frontiers in Physics, Edt. D. Pine (1990).

[17] Marc Sher and Yao Yuan, Phys. Rev. D, 44,(1991) 1461; Marc Sher, hep-ph/0006159v3; T.P. Cheng and M. Sher, Phys. Rev. D, 35 (1987) 3484.

[18] D. E. Groom,et. al., Particle Data Group, Eur. Phys. Jour. C,15 (2000)1 